\title{
Physical activity levels after treatment for breast cancer: two- year follow-up
}

An De Groef ${ }^{1}$, Inge Geraerts ${ }^{1}$, Heleen Demeyer ${ }^{2}$, Elien Van der Gucht ${ }^{1}$, Lore Dams ${ }^{3}$, Carlijn de Kinkelder ${ }^{4}$, Sanne Dukers - van Althuis ${ }^{4}$, Marijke Van Kampen ${ }^{1}$, Nele Devoogdt ${ }^{1,5}$

${ }^{1} \mathrm{KU}$ Leuven - University of Leuven, Department of Rehabilitation Sciences and University Hospitals Leuven, Department of Physical Medicine and Rehabilitation, Leuven, Belgium

${ }^{2} \mathrm{KU}$ Leuven - University of Leuven, Department of Rehabilitation Sciences and University Hospitals Leuven, Department of Respiratory Diseases, Leuven, Belgium; Support statement: $H D$ is a post-doctoral research fellow of the FWO-Flanders.

${ }^{3}$ Department of Rehabilitation Sciences and Physiotherapy, Faculty of Medicine and Health Sciences, University of Antwerp, Antwerp, Belgium

${ }^{4}$ Vrije Universiteit Brussel - Department of Physiotherapy, Physiology \& Anatomy, Faculty of Physical Education and Physiotherapy, Brussels, Belgium and European College for Lymphology \& Oncology, De Berekuyl/CSI-the Netherlands, the Netherlands.

${ }^{5}$ University Hospitals Leuven, Department of Vascular Surgery, Leuven, Belgium

For correspondence contact:

An De Groef, Physical Therapist, PhD

University of Leuven

Department of Rehabilitation Sciences

O\&N 4 Herestraat $49-$ bus 1510

3000 Leuven

Tel.: +3216342171

an.degroef@kuleuven.be 


\section{Abstract}

OBJECTIVES: Breast cancer treatment can have a considerable large and prolonged impact on activity levels of breast cancer survivors. Therefore, the aim of the present study was to investigate the change in total physical activity level and occupational, sport and household activity levels of breast cancer survivors from preoperatively up to 24 months after breast cancer surgery. Additionally, predictive factors for this change were investigated.

METHODS: Patients with primary breast cancer $(n=267)$ filled in the Flemish Physical Activity Computerized Questionnaire (FPACQ) before surgery and 1, 3, 6, 12 and 24 months after surgery. Patient-, disease- and treatment-related factors were analyzed as predictive factors for change in physical activity.

RESULTS: Two years after surgery, all activity levels (total, occupational, sport and household) were still significantly lower compared to preoperative values. After the first 12 months, no significant improvements were seen for none of the activity levels, except for the occupational activity.

CONCLUSIONS: Two years after breast cancer surgery, physical activity levels are still significantly lower compared to pre-operative values. Based on this limited recovery, it seems important to monitor physical activity levels in breast cancer patients and advice these patients to stay active after surgery and return to pre-operative activity levels in the long term as well. This study indicates the importance of long term monitoring and subsequently coaching of physical activity after breast cancer surgery.

KEYWORDS: Breast Neoplasms; Exercise; Activities of Daily Living 


\section{Introduction}

About $89 \%$ of women diagnosed with breast cancer survives five years after diagnosis ${ }^{1}$. This, among other things, is because of increasingly more and better options for cancer treatment ${ }^{1}$. However, several breast cancer treatment modalities such as breast and axillary surgery, radiotherapy, chemotherapy and hormone therapy can have a considerable large and prolonged negative impact on activity levels and quality of life ${ }^{2,3}$. Additionally, lower physical activity levels have also been shown to be associated with increased risk of breast cancer, worse disease outcome, more negative treatment related side effects, worse physical functioning and quality of life, higher risk of recurrence and eventually higher mortality ${ }^{4-10}$. Monitoring physical activity levels in breast cancer survivors is therefore of great importance, especially at long term.

Several studies, including one at our institution, indicated that one year after breast cancer surgery, pre-operative total physical activity level and occupational, sport and household activity levels are not yet recovered ${ }^{6,11-16}$. However, few studies have examined recovery of activity levels beyond this first year after breast cancer surgery, providing conflicting results. First, the study of Alfano et al showed that general physical activity levels reported at 24-months post-diagnosis were only slightly lower compared to (a retrospective report of) the pre-operative levels. Sixty-three percent of women reported maintaining or decreasing their sports and recreation activity during cancer treatment. Additionally, $65 \%$ of women reported to increase their household activity levels after the cancer diagnosis ${ }^{17}$. Mason et al concluded that recreational activity levels stayed low and relatively constant up to 5 years follow-up with no clear predictors for this ${ }^{18}$. Another study reported a recovery of activity levels from shortly after surgery to 1 year post-surgery, followed by a reduction in physical activity 5 years post-surgery ${ }^{13}$. Second, in addition to the reduced general and recreational physical activity levels, most women interrupt work activities (temporarily) during treatment ${ }^{19}$. The study of Islam et al concluded that $55 \%$ of breast cancer survivors returned to work after 12 months and $57 \%$ after 18 months ${ }^{20}$. The study of Roelen et al showed that $37 \%$ of absences lasted longer than one year and $12 \%$ of absences lasted longer than two years particularly in women aged 25-34 years. The mean duration before return to work was nearly a year ${ }^{21}$. Balak et al noticed as well that the mean duration before return to work was $11.4 \pm 5.5$ months and $35 \%$ of patients were absent longer than one year with $6 \%$ not returning to work within two years after diagnosis ${ }^{22}$.

In conclusion, recovery of the different levels of physical activity (occupational, sport and household) in breast cancer survivors beyond the first year after surgery differ among studies. In addition, to our knowledge, no study used preoperative levels to examine the trajectory of change of these different 
types of activity from preoperatively up to two years after surgery in the same population together. Further, in retrospective reports recall bias may influence the results ${ }^{17}$.

A wide range of predictive factors have been postulated for the change in activity levels at short term (i.e. up to one year after diagnosis). Patient-, disease- and treatment-related factors such as having a spouse, being employed, more invasive surgery, advanced age, active smoke behavior, higher prediagnosis activity level and higher Body Mass Index have been found to be associated with decreased physical activity levels one year after surgery ${ }^{6,11,12}$. To our knowledge, research into predictive factors for long term physical activity levels is very limited. One study reported a different recovery in general physical activity between women receiving chemotherapy versus women not receiving chemotherapy. Women receiving chemotherapy had an increase during the first 18 months after surgery and a decline afterwards. Women not receiving chemotherapy had a decline only after 3 years ${ }^{13}$.

Therefore, the first aim of the present study is to investigate the evolution of total physical activity level and occupational, sport and household activity levels of breast cancer survivors from preoperatively up to 2 years after breast cancer surgery. The second aim is to explore predictive factors for the changes in activity levels between the pre-operative stage and 2 years post-surgery. 


\section{Method}

This study is the two-year follow-up study of the longitudinal cohort study of $2010^{11}$. This study had approval from the Ethical Committee from University Hospitals Leuven (ML 3513). The design and procedure for this study have been previously described in the original article ${ }^{11}$. Important elements for the two-year follow-up study will be summarized here.

\section{Subjects}

Three hundred ninety-eight consecutive breast cancer patients treated in the Multidisciplinary Breast Center of the University Hospitals Leuven between September 2006 and September 2007, were asked to participate in this study. Inclusion criteria were women who had an axillary lymph node dissection or a sentinel node biopsy for a primary breast cancer. All patients that participated preoperatively were asked to participate again 2 years after surgery. Two hundred sixty-seven patients were available and were included in the two-year follow-up study.

\section{Procedure}

The day before surgery, breast cancer patients meeting the criteria were asked to participate in the study. If agreed to participate, patients filled in the Flemish Physical Activity Computerized Questionnaire (FPACQ) about their physical activities pre-operatively and 1, 3, 6, 12 and 24 months after surgery.

\section{Flemish Physical Activity Computerized Questionnaire (FPACQ)}

The FPACQ is a reliable and valid questionnaire. ${ }^{23}$ The questionnaire gives information on different physical activity variables: occupational status (employed or unemployed, working hours, job intensity and transport to the job), sport activities (three most frequently performed sports, frequency and duration of each sport) and household activity level (light, moderate and vigorous). Information about transport during leisure time and sedentary activities (TV and sleep) were also collected. Total physical activity level is the sum of occupational, sport, household activity levels and active transport in leisure time and sedentary activities. The activity variables were calculated with the Metabolic Equivalent Task (MET) values, which were determined using the Ainsworth compendium of activities ${ }^{24}$. Using the definition for a MET as the ratio of work metabolic rate to a standard resting metabolic rate of $1.0 \mathrm{~kg}^{-1} \mathrm{~h}^{-1}$, one MET is considered as the resting metabolic rate during quiet sitting. The calculations of each variable have been previously described in the original report ${ }^{11}$. The variables 
used in this research are total physical activity level with three sub-variables: occupational- sport- and household activity level.

\section{Statistical analysis}

A linear model for repeated measures was used to evaluate the evolution of various continuous variables (total, occupational, sport and household activity levels) over time. The model used an unstructured covariance matrix for measurements over time and has-compared to classical repeated measures ANOVA-the advantage that subjects with one or more missing measurements were still included in the analysis. The values were transformed (natural logarithm after adding a constant if necessary) to handle the right-skewed distribution of model residuals. Geometric means and $95 \%$ confidence intervals were obtained after transforming the means of the transformed variable to the original scale. Tukey adjustments were used for multiple comparisons between points in time. Notify that the analysis of the occupational activity level was restricted to pre-operatively employed patients $(n=145)$ and of the sport activity level to patients practicing some sport pre- operatively ( $n=144)$.

Further, the predictive value of patient- related factors (spouse (yes/no), employment status (unemployed/employed/retired), smoking (yes/no)), disease-related factors (lymph node stage (pNO/pN1/pN2-3), type of breast cancer (ductal/other)) and treatment-related factors (type of axillary surgery (sentinel biopsy/axillary dissection), number of lymph nodes dissected $(<10 / 10-19 />19)$, chemotherapy (yes/no)) for the change in total, occupational, sport and household activity levels between the pre-operative stage and 24 months after surgery were analyzed. For this purpose, parametric tests (Independent T test, ANOVA and Pearson correlation) were performed on the sample of subjects with data after 24 months ( $n=209)$, the aim being to study the association between these characteristics and the observed change (=bi-variable analyses). General linear models were used to perform multi-variable analyses.

Results should be interpreted with care given the multitude of models and P values. All analyses were performed using SAS software, version 9.4 of the SAS System for Windows and SPSS 24.0, $p$ value $<0.05$ was considered statistically significant. 


\section{Results}

Two hundred sixty-seven patients filled in FPACQ before surgery, 242 (91\%), 243 (91\%), 234 (88\%), 236 $(88 \%)$ and 209 (78\%) at 1, 3, 6, 12 and 24 months, respectively. Baseline characteristics of the included and excluded breast cancer patients can be found in table $1^{11}$. Age of included patients ranged between 21 and 90 years, with a mean of 55 years. Eighty patients were retired, 145 were employed and 42 patients were unemployed before surgery.

Table 1. Characteristics of the excluded $(n=131)$ and included $(n=267)$ breast cancer patients.

\begin{tabular}{|c|c|c|c|c|}
\hline \multicolumn{5}{|c|}{ Breast Cancer Patients } \\
\hline & Excluded $(n=131)$ & Included ( $n=267)$ & & $P$ \\
\hline Age (year) & $64(13)$ & $55(11)$ & $<0.01$ & \\
\hline $\begin{array}{l}\text { Body Mass Index } \\
\left(\mathrm{kg} / \mathrm{m}^{2}\right)\end{array}$ & $27.5(5.1)$ & $25.1(4.4)$ & 0.02 & \\
\hline \multicolumn{5}{|l|}{ Gender } \\
\hline Women & 130 (99\%) & 265 (99\%) & 0.96 & \\
\hline Men & $1(1 \%)$ & $2(1 \%)$ & & \\
\hline \multicolumn{5}{|l|}{ Tumour Stage } \\
\hline Tis & $8(6 \%)$ & $7(3 \%)$ & 0.94 & \\
\hline $\mathrm{T} 1$ & $52(40 \%)$ & $123(46 \%)$ & & \\
\hline $\mathrm{T} 2$ & $59(44 \%)$ & $107(40 \%)$ & & \\
\hline T3 & $9(7 \%)$ & $18(7 \%)$ & & \\
\hline T4 & $3(2 \%)$ & $12(5 \%)$ & & \\
\hline \multicolumn{5}{|l|}{ Lymph Node Stage } \\
\hline NO & $81(62 \%)$ & $154(58 \%)$ & 0.56 & \\
\hline N1 & $38(29 \%)$ & $93(35 \%)$ & & \\
\hline N2 & $8(6 \%)$ & $13(5 \%)$ & & \\
\hline N3 & $4(3 \%)$ & $7(2 \%)$ & & \\
\hline \multicolumn{5}{|c|}{ Type of Breast Surgery } \\
\hline Mastectomy & $64(49 \%)$ & $119(45 \%)$ & 0.42 & \\
\hline Brest conserving & $67(51 \%)$ & $148(55 \%)$ & & \\
\hline $\begin{array}{l}\text { Type of Axillary } \\
\text { Surgery }\end{array}$ & & & & \\
\hline
\end{tabular}




\begin{tabular}{llll} 
Axillary Dissection & $92(70 \%)$ & $158(59 \%)$ & 0.03 \\
\multicolumn{1}{c}{ Sentinel Biopsy } & $39(30 \%)$ & $109(41 \%)$ & \\
Radiotherapy & $106(81 \%)$ & $231(87 \%)$ & 0.15 \\
Chemotherapy & $52(40 \%)$ & $137(51 \%)$ & 0.03 \\
Hormonal therapy & $102(78 \%)$ & $213(80 \%)$ & 0.66
\end{tabular}

Mean (SD) and numbers (\%) are given for continuous and categorical data, respectively. $P$ values form Independent $\mathrm{t}=$ test and $\mathrm{X}^{2}$ test.

\section{Evolution of total physical activity}

Total activity level of the breast cancer patients decreased from 270 (27) MET-hours/week before surgery to 257 (26) MET-hours/week after 12 months and 260 (26) MET-hours/week at 24 months (Figure 1). At 24 months follow-up, total activity level was still significantly lower than before surgery $(p<0.001)$. Remarkably, total activity level increased significantly between each time point except from 12 months up to 24 months $(p=0.3041)$.

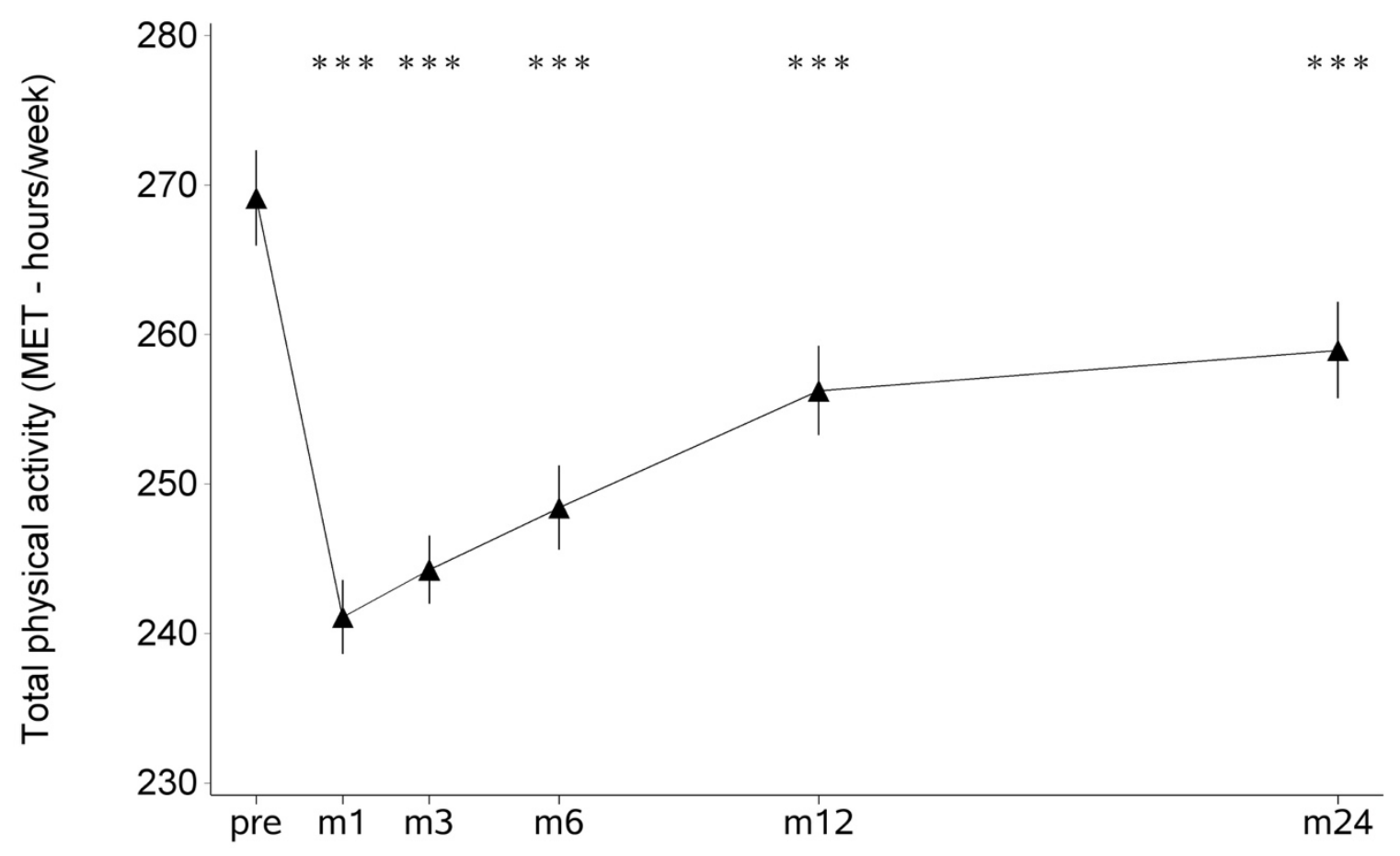

Figure 1: Evolution of total physical activity level of breast cancer patients $(n=267)$ from preoperatively to 24 months after surgery. Whiskers refer to 95\% Confidence Interval for the (geometric) mean.

Evolution of occupational physical activity level 
For occupational activity level, a similar trend was found. For breast cancer patients employed before surgery ( $n=145)$, the occupational activity level decreased from 79 MET-hours/week before surgery to 12 and 21 MET-hours/week at 12 and 24 months follow-up, respectively (figure 2). At each follow-up, occupational activity level was significantly lower than preoperatively $(p<0.001)$. A significant increase in occupational activity level was found between each time point, including from 12 to 24 months $(p=0.0028)$.

When looking at the breast cancer survivors who were employed before surgery, only $60 \%$ had returned to work 12 months post-surgery. At 24 months post-surgery, $74 \%$ of patients had gone back to work. Remarkably, both at 12 and 24 months post-surgery only $34 \%$ of employed breast cancer patients worked full time, compared to $43 \%$ before surgery.

\section{Evolution of sports physical activity level}

Looking at all patients performing some sport postoperatively, MET-hours/week decreased from 13 MET-hours/week before surgery to 8 and 7 MET-hours/week at 12 and 24 months post-surgery, respectively. At these time points, sport activity level was still significantly lower compared to baseline $(p<0.001)$. Remarkably, between time points, only between 6 and 12 months post-surgery a significant increase in sport activity level was noticed $(p<0.001)$ (figure 2 ).

When looking at the breast cancer survivors who were sport-active before surgery, $78 \%$ practiced some sport between 1 and 12 months after surgery. Up to 24 months, this number remained $72 \%$ of the pre-operatively sport-active patients. For patients not performing a sport before surgery, $16 \%$ and $28 \%$ reported performing a sport at 12 and 24 months, respectively.

Evolution of household physical activity level

At last, household activity levels decreased from 35 MET-hours/week to 29 and 27 MET-hours/week after 12 and 24 months, respectively. Activity levels were still significantly lower compared to preoperatively levels $(p<0.001)$. When looking at changes between time points, a significant increase in activity levels was noticed only between 1 and 3 months post-surgery $(p<0.001)$. 


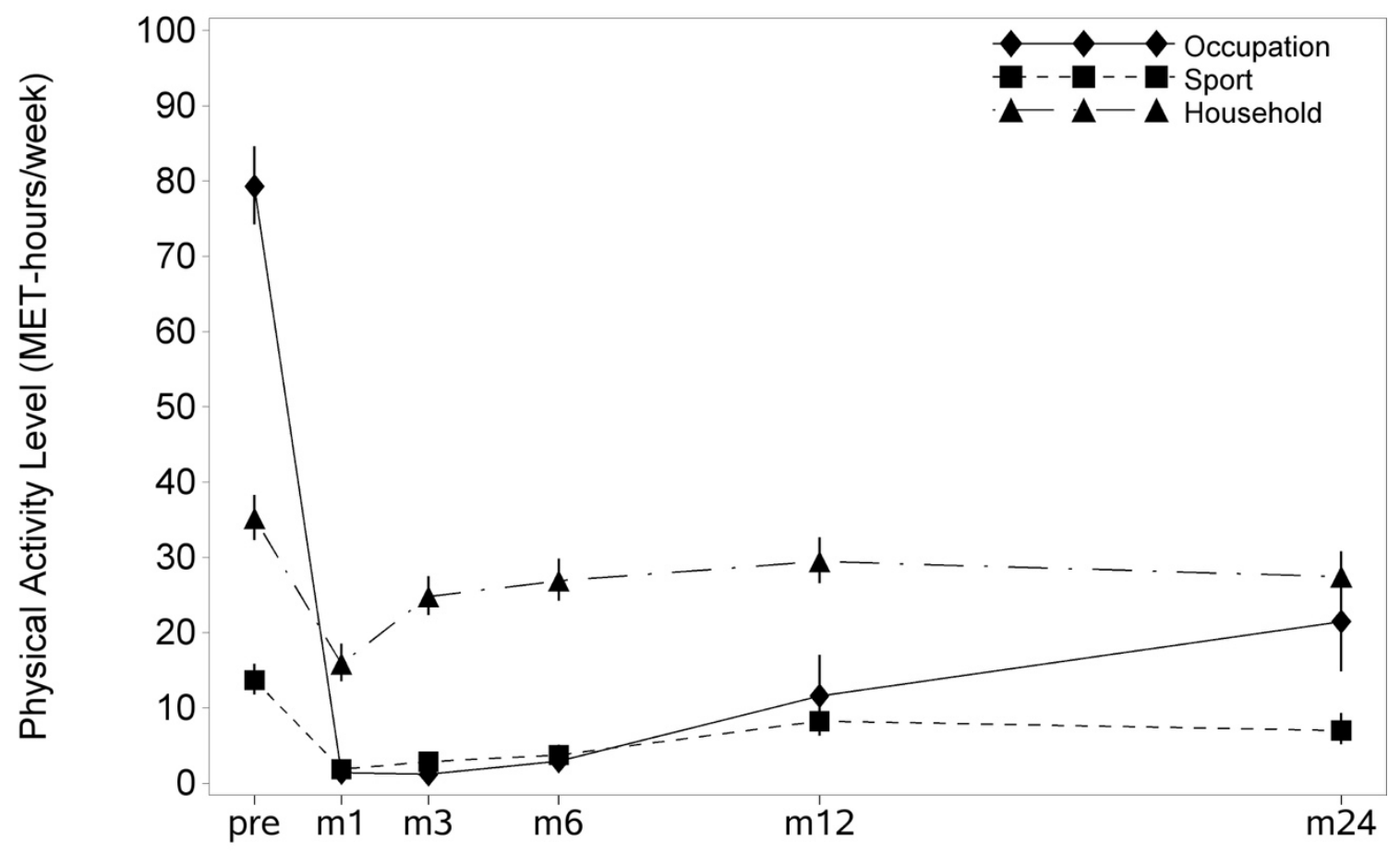

Figure 2: Evolution of the occupational ( $n=145)$, sport $(n=144)$ and household activity level $(n=267)$ of breast cancer patients from preoperatively to 24 months post-surgery. Whiskers refer to 95\% Confidence Intervals for the (geometric) mean.

\section{Predictive factors for changed physical activity levels}

Bi-variable and multivariable analyses are performed similar as in the one-year follow up study ${ }^{11}$. The dependent variable is the change in activity levels between the preoperative stage and 24 months post-surgery.

In the bivariable analysis, most significant predictors for change in the different physical activity levels between the preoperative stage and 12 months after surgery were no longer significantly associated. Only 'having a spouse' and 'undergoing chemotherapy' remained predictive for a greater decrease in occupational activities. One remarkably new association for the change in occupational activity over 2 years is a significant correlation between age and change in occupational activity $(r=0.275 ; p<0.001)$. This result indicates that younger patients had a greater decrease in occupational activity level than older patients. This was not found 12 months post-surgery. Comparable with the one year follow-up study, older patients still have a higher risk of having a decreased sport activity level 24 months after surgery.

For the multivariable analysis, similar as for the one-year follow up study, higher lymph node stage remained a significant predictor for a greater decline in occupational activity level $(p=0.011)$. One new significant association was found between chemotherapy and household activity level. Having no 
chemotherapy is predictive for a larger decrease in household activities (-11 MET-hours/week, $p=0.009$ ). 


\section{Discussion}

Results of the present study indicate that none of the activity levels (total, occupational, sport and household) reached preoperative values 2 years post-surgery in breast cancer survivors. More specific, from 1 to 2 years post-surgery no additional increase was found, except for occupational activity level.

In the present study, total physical activity level of breast cancer survivors was still significantly lower 24 months post-surgery compared to pre-operatively. There was no significant change between 12 and 24 months post-surgery. Other studies confirm that activity levels are not recovered at long term. The study of Alfano et al ${ }^{17}$ showed that total physical activity levels reported at 24-months postdiagnosis were lower compared to pre-operative levels. However, they did not report significance levels. Mason et al concluded that up to two years after diagnosis total physical activity levels stayed constant over time. However, they did not compare these results to pre-operative levels but to six months after diagnosis ${ }^{18}$.

Similar to total physical activity level, occupational activity levels were significantly lower 12 and 24 months after surgery compared to pre-operative values. However, from 12 to 24 months post-surgery, both the occupational activity level did increase significantly and the percentage of breast cancer survivors that returned to work (60\% and $74 \%$ at 12 and 24 months, respectively). In other studies, numbers vary between $57 \%$ and $78 \%$ of patients who returned to work up to 24 months post-surgery, with a mean work absence reported around 1 year. ${ }^{11,20-22}$ These differences in duration of sick leave probably depend on the different countries and health care systems. More insight is needed in the factors that contribute to the return to work in breast cancer patients, so that the different results can be explained and targeted monitoring can be developed.

Both at 12 and 24 months post-surgery a decrease in sport activity level was seen compared to preoperatively. Despite the lower activity level, a considerably large amount of breast cancer survivors reported to be sport-active in our study. Seventy-two percent of the pre-operatively sport-active patients are performing some sports at 24 months. In addition, $28 \%$ of the patients not performing sports pre-operatively were sport-active at 24 months as well. However, Mason et al ${ }^{18}$ showed that 24 months after surgery only $40 \%$ of breast cancer survivors met the guidelines of physical activity at 24 months. In our study, mean MET-hours/week for sport was only 7, which is also below the recommendations of 10 MET-hours/week by the World Health Organization.

At 12 and 24 months, household activity levels were significantly decreased compared to preoperatively. No significant difference was found between 12 and 24 months. This contrasts with the study of Alfano. ${ }^{17}$ They reported that $65 \%$ of women increased their activity levels across the cancer 
experience, but they reported no significance levels. Remarkably, in the present study, a significant increase in household activity level was seen only between 1 and 3 months post-surgery. Afterwards the household activity level remained stable.

Patient-, disease- and treatment- related factors found to be associated with the change in activity levels up to 1 year after surgery were either the same or no longer significantly associated with the change between preoperative physical activity levels and 24 months post-surgery. Chemotherapy appears to be an important predictive factor for occupational activity and household activity, however in opposite direction. Receiving chemotherapy had a negative influence on recovery of occupational activity which can be probably explained by a longer active treatment schedule and more prolonged side effects and complications compared to not receiving chemotherapy. For the relationship between not receiving chemotherapy and a larger decrease in household activity at 2 years a clear explanation is not available. Interpreting these results, the chance of a false positive results should be taken into account given the multiple testing.

The results of the present study have important clinical implications. The results reflect the importance of monitoring physical activity of breast cancer survivors also beyond the first year post-surgery. Many hospitals offer rehabilitation and recovery programs during active treatment but not afterwards. Promoting physical activity after this period may be necessary as well for returning to preoperative values. In the present study, the focus was on association between patient- disease- and treatmentrelated factors and change in physical activity. Given the limited significant associations, other factors should be taken into account for recovery of physical activity. As indicated by the study of Emery et al, psychosocial and behavioral factors may be of more influence on recovery of physical activity in breast cancer survivors and should be monitored and coached from early postoperative as well ${ }^{13,25}$.

The present study has several strengths. First, preoperative physical activity levels were available. By measuring PA one day before the surgery, an accurate baseline assessment of physical activity was obtained, a limitation of previous research in the same area. Second, this is the first long-term study assessing all aspects of physical activity (occupational, sport and household activities, transport during leisure time and sedentary activities) in the same population. Additionally, the present study is based on a large sample of whom a considerably large group of women completed the assessments at two years post-surgery. A limitation may be that the FPACQ is subjective measurement method of physical activity. Studies have indicated that the FPACQ may lead to an overestimation of the physical activity level. ${ }^{26}$ Nevertheless, this will not have affected the within patient comparisons of the present study. Second, patients included in the analyses differed from those not included for some patient (age, BMI) 
and treatment (chemotherapy) related characteristics. This could have affected the external validity of the results.

Further research should explore why physical activity levels only partly recover after surgery. Better monitoring of return to work and activity coaching interventions may enhance this. Additionally, it would also be interesting to investigate if the physical activity levels pre-operatively are in fact comparable to healthy persons. Knowing that physical inactivity is an important predictor for having breast cancer, it could be hypothesized that pre-operative levels are below those of healthy controls. At last, as mentioned in the limitation section, the FPACQ is a subjective measurement of physical activity. ${ }^{23}$ Studies with more objective measurement methods should be conducted to have a more realistic view on the evolution of physical activity after breast cancer surgery. First, accelerometry measurements such as The Actigraph GT3X (Actigraph LLC, Pensacola, FL, USA), and DynaPort MoveMonitor (McRoberts BV, The Hague, the Netherlands) are validated activity monitors that should give more accurate information. ${ }^{27,}{ }^{28}$ Second, consumer-wearable activity trackers (e.g. Fitbit) have become more popular. ${ }^{29}$ Validity and reliability of these devices should be further investigated but they may be more objective compared to questionnaires. Additionally, these user-friendly devices have the advantage that they can also be used to coach patients. ${ }^{29}$

In conclusion, none of the different activity levels returned to pre-operative values after 2 years. Additionally, it is remarkable that between 12 and 24 months post-surgery no significant increases were found for the different activity levels either. Only for occupational activity level a significant in increase in MET-hours/week was found. This study indicates the importance of long term monitoring and subsequently coaching of physical activity after breast cancer surgery. 


\section{Conflict of interest}

No conflict of interest

\section{Funding source}

This study was supported by a grant from the agency for Innovation by Science and Technology (Applied Bio- medical Research). The authors declare that they have no other conflicts of interest.

\section{Ethical approval}

This study had approval from the Ethical Committee from University Hospitals Leuven (ML 3513). 


\section{References}

1. Ferlay J, Soerjomataram I, Dikshit R, et al. Cancer incidence and mortality worldwide: sources, methods and major patterns in GLOBOCAN 2012. International journal of cancer Journal international du cancer 2015; 136: E359-386. 2014/09/16. DOI: 10.1002/ijc.29210.

2. Penttinen HM, Saarto T, Kellokumpu-Lehtinen $P$, et al. Quality of life and physical performance and activity of breast cancer patients after adjuvant treatments. Psycho-oncology 2011; 20: 1211-1220. 2010/09/30. DOI: 10.1002/pon.1837.

3. Hidding JT, Beurskens $\mathrm{CH}$, van der Wees PJ, et al. Treatment related impairments in arm and shoulder in patients with breast cancer: a systematic review. PloS one 2014; 9: e96748. 2014/05/13. DOI: 10.1371/journal.pone.0096748.

4. Phillips SM, Dodd KW, Steeves J, et al. Physical activity and sedentary behavior in breast cancer survivors: New insight into activity patterns and potential intervention targets. Gynecologic oncology 2015; 138: 398-404. 2015/06/01. DOI: 10.1016/j.ygyno.2015.05.026.

5. $\quad$ Ottenbacher AJ, Day RS, Taylor WC, et al. Long-term physical activity outcomes of homebased lifestyle interventions among breast and prostate cancer survivors. Supportive care in cancer : official journal of the Multinational Association of Supportive Care in Cancer 2012; 20: 2483-2489. 2012/01/18. DOI: 10.1007/s00520-011-1370-y.

6. Irwin ML, Crumley D, McTiernan A, et al. Physical activity levels before and after a diagnosis of breast carcinoma: the Health, Eating, Activity, and Lifestyle (HEAL) study. Cancer 2003; 97: 17461757. 2003/03/26. DOI: 10.1002/cncr.11227.

7. Fassier P, Zelek L, Partula V, et al. Variations of physical activity and sedentary behavior between before and after cancer diagnosis: Results from the prospective population-based NutriNetSante cohort. Medicine 2016; 95: 10. Article. DOI: 10.1097/md.0000000000004629.

8. Branstrom R, Petersson LM, Saboonchi F, et al. Physical activity following a breast cancer diagnosis: Implications for self-rated health and cancer-related symptoms. European Journal of Oncology Nursing 2015; 19: 680-685. Article. DOI: 10.1016/j.ejon.2015.04.008.

9. Campbell KL, Pusic AL, Zucker DS, et al. A prospective model of care for breast cancer rehabilitation: function. Cancer 2012; 118: 2300-2311. DOI: 10.1002/cncr.27464.

10. Kyu HH, Bachman VF, Alexander LT, et al. Physical activity and risk of breast cancer, colon cancer, diabetes, ischemic heart disease, and ischemic stroke events: systematic review and doseresponse meta-analysis for the Global Burden of Disease Study 2013. BMJ (Clinical research ed) 2016; 354. 10.1136/bmj.i3857.

11. Devoogdt N, Van Kampen M, Geraerts I, et al. Physical activity levels after treatment for breast cancer: one-year follow-up. Breast cancer research and treatment 2010; 123: 417-425. 2010/06/29. DOI: 10.1007/s10549-010-0997-6.

12. Littman AJ, Tang MT and Rossing MA. Longitudinal study of recreational physical activity in breast cancer survivors. Journal of cancer survivorship : research and practice 2010; 4: 119-127. 2010/02/25. DOI: 10.1007/s11764-009-0113-2.

13. Emery CF, Yang HC, Frierson GM, et al. Determinants of physical activity among women treated for breast cancer in a 5-year longitudinal follow-up investigation. Psycho-oncology 2009; 18: 377-386. 2009/02/26. DOI: 10.1002/pon.1519.

14. Pinto BM, Trunzo JJ, Reiss $P$, et al. Exercise participation after diagnosis of breast cancer: trends and effects on mood and quality of life. Psycho-oncology 2002; 11: 389-400. 2002/09/14. DOI: 10.1002/pon.594.

15. Andrykowski MA, Beacham AO and Jacobsen PB. Prospective, longitudinal study of leisuretime exercise in women with early-stage breast cancer. Cancer epidemiology, biomarkers \& prevention : a publication of the American Association for Cancer Research, cosponsored by the American Society of Preventive Oncology 2007; 16: 430-438. 2007/03/21. DOI: 10.1158/10559965.epi-06-0735. 
16. Harrison S, Hayes SC and Newman B. Level of physical activity and characteristics associated with change following breast cancer diagnosis and treatment. Psycho-oncology 2009; 18: 387-394. 2009/01/02. DOI: 10.1002/pon.1504.

17. Alfano $\mathrm{CM}$, Smith $\mathrm{AW}$, Irwin $\mathrm{ML}$, et al. Physical activity, long-term symptoms, and physical health-related quality of life among breast cancer survivors: a prospective analysis. Journal of cancer survivorship : research and practice 2007; 1: 116-128. 2008/07/24. DOI: 10.1007/s11764-007-0014-1. 18. Mason C, Alfano CM, Smith AW, et al. Long-term physical activity trends in breast cancer survivors. Cancer epidemiology, biomarkers \& prevention : a publication of the American Association for Cancer Research, cosponsored by the American Society of Preventive Oncology 2013; 22: 11531161. 2013/04/12. DOI: 10.1158/1055-9965.epi-13-0141.

19. Feuerstein M, Todd BL, Moskowitz MC, et al. Work in cancer survivors: a model for practice and research. Journal of cancer survivorship : research and practice 2010; 4: 415-437. 2010/10/15. DOI: 10.1007/s11764-010-0154-6.

20. Islam T, Dahlui M, Majid HA, et al. Factors associated with return to work of breast cancer survivors: a systematic review. BMC Public Health 2014; 14 Suppl 3: S8. 2014/12/02. DOI: 10.1186/1471-2458-14-s3-s8.

21. Roelen CA, Koopmans PC, de Graaf JH, et al. Sickness absence and return to work rates in women with breast cancer. International archives of occupational and environmental health 2009; 82: 543-546. 2008/09/17. DOI: 10.1007/s00420-008-0359-4.

22. Balak F, Roelen CA, Koopmans PC, et al. Return to work after early-stage breast cancer: a cohort study into the effects of treatment and cancer-related symptoms. Journal of occupational rehabilitation 2008; 18: 267-272. 2008/08/02. DOI: 10.1007/s10926-008-9146-z.

23. Matton L, Wijndaele K, Duvigneaud N, et al. Reliability and validity of the Flemish Physical Activity Computerized Questionnaire in adults. Research quarterly for exercise and sport 2007; 78: 293-306. 2007/10/19. DOI: 10.1080/02701367.2007.10599427.

24. Ainsworth BE, Haskell WL, Herrmann SD, et al. 2011 Compendium of Physical Activities: a second update of codes and MET values. Medicine and science in sports and exercise 2011; 43: 15751581. 2011/06/18. DOI: 10.1249/MSS.0b013e31821ece12.

25. Charlier C, Van Hoof E, Pauwels E, et al. Treatment-related and psychosocial variables in explaining physical activity in women three weeks to six months post-treatment of breast cancer. Patient Educ Couns 2012; 89: 171-177. 2012/07/31. DOI: 10.1016/j.pec.2012.06.029.

26. Scheers T, Philippaerts R and Lefevre J. Assessment of physical activity and inactivity in multiple domains of daily life: a comparison between a computerized questionnaire and the SenseWear Armband complemented with an electronic diary. The international journal of behavioral nutrition and physical activity 2012; 9: 71. 2012/06/14. DOI: 10.1186/1479-5868-9-71.

27. Rabinovich RA, Louvaris $Z$, Raste $Y$, et al. Validity of physical activity monitors during daily life in patients with COPD. The European respiratory journal 2013; 42: 1205-1215. 2013/02/12. DOI: 10.1183/09031936.00134312.

28. Dowd KP, Szeklicki R, Minetto MA, et al. A systematic literature review of reviews on techniques for physical activity measurement in adults: a DEDIPAC study. The international journal of behavioral nutrition and physical activity 2018; 15: 15. 2018/02/10. DOI: 10.1186/s12966-017-06362.

29. Evenson KR, Goto MM and Furberg RD. Systematic review of the validity and reliability of consumer-wearable activity trackers. The international journal of behavioral nutrition and physical activity 2015; 12: 159. 2015/12/20. DOI: 10.1186/s12966-015-0314-1. 